\title{
Managing incidental findings in population based biobank research
}

\author{
Berge Solberg and Kristin Solum Steinsbekk \\ NTNU, Det medisinske fakultet, Institutt for samfunnsmedisin, Postboks 8905, MTFS, 7491 Trondheim \\ E-mail: berge.solberg@ntnu.no
}

\begin{abstract}
With the introduction of whole genome sequencing in medical research, the debate on how to handle incidental findings is becoming omnipresent. Much of the literature on the topic so far, seems to defend the researcher's duty to inform, the participant's right to know combined with a thorough informed consent in order to protect and secure high ethical standards in research. In this paper, we argue that this ethical response to incidental findings and whole genome sequencing is appropriate in a clinical context, in what we call therapeutic research. However, we further argue, that it is rather inappropriate in basic research, like the research going on in public health oriented population based biobanks. Our argument is based on two premises: First, in population based biobank research the duties and rights involved are radically different from a clinical based setting. Second, to introduce the ethical framework from the clinical setting into population based basic research, is not only wrong, but it may lead to unethical consequences. A Norwegian population based biobank and the research-ethical debate in Norway on the regulation of whole genome sequencing is used as an illustrative case to demonstrate the pitfalls when approaching the debate on incidental findings in population based biobank research.
\end{abstract}

An incidental finding in medical research is defined as "a finding concerning an individual research participant that has some potential health or reproductive importance and is discovered in the course of conducting research but is beyond the aims of the study" [1]. Although incidental findings appear in a lot of different research projects where various medical technologies are used, for instance MRI, the hottest debates have taken place with regard to genetics and genomics in general, and whole genome sequencing in particular. Sequencing of whole genomes is just now becoming technologically and economically feasible in medical research and the problem of "knowing too much,"as Hans Jonas once talked about [2], has to be dealt with on a very practical level. Incidental findings seem to be an inevitable part of a new research landscape and how to handle this needs to be thoroughly thought through. The purpose of this paper is to revisit the moral and ethical relation between researchers and research participants in genomic research. We will use two different cases to explore the challenges of incidental findings when whole genome sequencing or related technologies are being used. These two scenarios, a clinical context and one in basic research, gives the analysis important depth and nuances since they represent two extremities within human genome research.

\section{CliniCAl Rights AND DUTIES}

To open up the moral landscape of incidental findings, we should start in the clinical context. This is a setting most of us are familiar with and we have rather clear and articulated intuitions and beliefs on what is right and wrong here. A central feature of what we will call the clinical context is for instance that there is a doctor and there is a patient where a doctor-patient relationship exists. These factors have some ethical implications. The patient exists because a person has contacted the health care system in order to get help for some kind of disease, symptoms or medical related anxiety. An intention to get help exists. At the same time there exists a doctor, a physician, whose primary training and intention is to give help, in the sense that he or she has the competence, the will and the obligation to diagnose, to reduce suffering, to heal and even to communicate - in a good way.

When the doctor and the patient meet we have a doctor-patient-relation. This relation is filled with ethical aspirations - the relation should be a relation of mutual respect, there should be openness, empathy and trust. The ethical aspirations are backed with juridical norms; in most countries the doctor has a duty to inform the patient about her condition (to a certain degree the moral duty to tell the truth), the patient has a corresponding right to know, the doctor has a duty not to harm the patient as well as a duty to inform the patient and thereby respect patient autonomy.

In a sense, incidental findings have always been part of the clinical context. A patient may have entered the hospital because of kidney problems. During the examination the doctors incidentally discover a tumor unrelated to the patients initial problems. In such a setting, there is not much of a discussion on a "right not to know" or a non-disclosure policy. The established ethical and juridical norms tell us that the doctor's obligation is to inform the patient and thereby respond 
to the patient's intention to get to know his or her medical condition (to get well/treated). The patient has a right to know, based on the premise that the patient is already there, in the clinical context, in order to know. These duties can be argued to extend into genomic research when research is done in clinical contexts. This follows Ravitsky and Wilfond (2007) arguments for disclosure of genomic information to research participants, for example in longitudinal family studies of serious congenital disease where often more than one member of the family are affected [3]. In cases like these there are ongoing involvement between the patient(s), his or her family and the health care system.

\section{RESEARCH ETHICAL RIGHTS AND DUTIES}

When we change the context from a clinical one to a context of basic research - like population based biobanking - ethical rights and duties, we claim, change too. The patient, the doctor and the doctor-patient relation are all absent. Of course, there will be patients involved in population based biobank research. Indeed there will be doctors involved as well. Therefore it seems like doctor-patient relationships might exist. But it does not, because in these settings the patient is primarily a participant and the doctor is primarily a researcher, like any other non-patients and non-doctors taking part. The participant has not attended the biobank in order to be helped or healed. The researcher is not part of research biobanking in order to treat actual participants. They both take part in this type of endeavor to contribute to research - to future knowledge production. The hope is that this knowledge can in part build the foundation on which future diagnostics, treatments and preventions will stand. There does not seem to exist any kind of relation between the participant and the researcher which resembles the doctor-patient relation. The fundamental premise for a right to know, and a corresponding duty to inform are absent from this context.

What if a participant believes that he or she is to gain therapeutically from participating in population based biobank research? And what if a researcher believes that he or she has to return incidental findings in order to let individual participants gain therapeutically from research? As many commentators have claimed, offering individualized information to research participants invites them, as well as researchers and review boards, into the therapeutic misconception [4-7]. The therapeutic misconception is characterized by a misunderstanding of the difference between research and treatment. The researcher's primary goal is not, and cannot be, to benefit any one participant [4]. If you, as a participant, believe so, you are taking part in most research on wrong premises. Population based biobank participants are in Norway as in several other countries invited into these projects on the premise of no return of individual genetic findings or results. While they of course hope that the research will inevitably help them if help is needed - it is for the benefit of us as a collective, for future patients as a group, in which participation is framed.

Moving from the clinical context to a context of population based biobanking, it seems that we cannot just transfer the clinical ethos to the research setting. The contexts are different in an ethically relevant way. The central premises for informational rights and duties in the clinical context are absent in the research context. However, even though we cannot base a duty to disclose on for instance a doctor-patient relation, it is of course possible that a duty to disclose can be based on other relations or premises. Reciprocity, justice, respect as well as beneficence seem to be relevant candidates for basing a duty to disclose individual results in research, as suggested by Ravitsky and Wilfond [3].

\section{RECIPROCITY, JUSTICE, RESPECT AND BENEFICENCE}

Notions of reciprocity, justice, respect and beneficence all seem to capture something essential in the relation between researchers and research participants. A research participant gives something of value to the researchers. Isn't it then both respectful and just that the researchers return results of potential value to the individual participants, the argument goes. Why shouldn't researchers have a duty to benefit participants when research benefits from the participants contributions? Doesn't reciprocity in the participant-researcher relation support a duty to disclose?

There is no doubt that research and researchers should be concerned with beneficence. Anticipated beneficence is what justifies that research participants are exposed to a certain risk of physical or informational harms, burdens and inconveniences. Further, anticipated beneficence justifies the use of research funding. Without any prospect of beneficence, research should not be conducted. The important question, however, is towards whom we should think that beneficence should be directed in research - the actual individual research participant or the (future) general population. We will argue for the latter. Any medical research project, independent of whether individual results are returned or not, should meet the requirements of beneficence. This means, as Meltzer has indicated, that "a research project must meet the requirements of beneficence regardless of whether it returns individual research results" [4]. The requirement of beneficence has to be understood on a collective level, not on an individual level. And if the requirements of beneficence in research must be met independently of possible return of results, then "it cannot be the case that the requirements of beneficence require investigators to offer results" [4].

Still, even though the requirements of beneficence do not require investigators to offer individual results, research participants must be respected. They should not be left with a feeling of being exploited or becoming worse off as a consequence of participating in 
research. However, as Parker has argued, "incidental findings that are not disclosed to the participant, and that are safeguarded from third-parties, present little risk of making a subject worse-off" [6]. Further, as Ossorio argues, "not to disclose individual results does not mean treating participant like mere means (in the Kantian sense). Individuals who understand the information provided in nondisclosure policies of a research protocol, and who nonetheless choose to participate, are treated as an end in themselves" [9]. As we see, equating a non-disclosure policy with disrespect or exploitation of research participants can be refuted. Still, in order to fully capture what respectful treatment of participants in biobank research entails, we need to change our perspective and look at this from the logic of research participation and resource utilization, as suggested by Forsberg et al [10].

\section{RESPECTING PARTICIPANTS BY MAXIMIZING RESEARCH}

Research ethics, according to a common sense view, is about protecting the welfare of individual research participants while he or she is taking part in research. A central point in the Helsinki declaration is that the welfare of the individual must take precedence over all other interests [11]. Although this is an important research ethical insight based on historical lessons, it might nevertheless have an unwanted side effect. The focus on the individual - an individual in need of protection from a society that might harm may in fact stimulate an unhealthy individualism in research ethics. Research is inherently a collective undertaking which might be restricted or even hampered if ethics blind us from acknowledging that individuals as part of the society, in which we live, may have interest that are of a collective nature.

Donating a blood sample to large-scale biobank research has been compared to donating money to a good cause like the Red Cross. What does Red Cross owe a person who has donated a smaller or larger sum of money to their activities? How does Red Cross show the donor respect? When Forsberg et al made this analogy, they stressed the following point: "The only moral duty the Red Cross has, from the donor's point of view, is to make sure that the donation leads to as much of the intended good as possible"[10]. Respect for the donor, in this perspective, means to use the donation or the sample for the intended purpose. Respect for the donor means maximizing the benefit of the donation. Therefore to favour a system of disclosure of individual research results at the expense of research outcomes is to disrespect the individual participant.

The last point can be made clearer by taking the analogy with Red Cross even further. Among charitable institutions it has become increasingly popular to give feedback to the donors about ongoing projects and the results of such projects in newsletters, magazines and so on. On the one hand, this is interesting to read; it may create larger engagement, encourage involvement, give oversight and a form of control and thereby contribute to increased recruitment. On the other hand, such services create a bureaucracy and engage more and more people and resources in activities that do not necessarily support the core activity of the Red Cross. Obviously the balance between resources spent on follow up of individual donors and resources spent on the collective purpose can tip the wrong way. If there is a tension here, there is no doubt where the moral duty towards the donor lies: Respecting the donor means using the donated money for the intended purpose [10].

A central question for population based biobanking, then, is to answer whether there is a real tension between the intended purpose of biobanking, research, and return of incidental findings to individual participants. Does returning such findings to individual participants in population based biobanking in any way stand in opposition to the intended purpose of the research? In order to answer that question we need to take a look into a real case of population based biobanking.

\section{Possible haRMful CONSEQUENCES OF A DISCLOSURE POLICY}

In Norway all medical research is regulated under the Norwegian Health Research Act [12]. In rare circumstances the Norwegian Biotechnology Act may apply to medical research, especially in cases where genetic results are planned to be returned [13]. While no consensus has been reached so far, some of the members of the Norwegian Advisory Board on Biotechnology suggested that whole genome sequencing in biobanks must be subjugated to the Biotechnology Act, and that the non-disclosure policy must be reconsidered [14]. Here we take a short look on how such a change of policy might affect one of the largest biobanks of Norway, the HUNT biobank.

The HUNT study was founded in the 1970's of a politically radical movement of doctors with a strong interest in public health and a clear agenda to fight "medicalization" in society [15]. This critical public health mission has been a central focus in several publications from HUNT, for instance illustrated by research on the effects of implementation of the European guidelines on cardiovascular disease prevention in clinical practice [16]. HUNT Biobank was added to the HUNT study in the mid 90's. Today it contains more than 250,000 samples of human DNA. The HUNT study and the HUNT Biobank have followed a non-disclosure policy. All research projects use coded samples and data, therefore researchers, using HUNT resources, do not know the identity of participants involved in the projects. In addition all projects have a public health focus, and the identity of individual research participants have no bearing or value for the research outcomes.

Generally, a disclosure-policy can in principle mean everything from returning a file of raw data from whole 
genome sequencing (all the rows of A,T,C and G's) to a carefully quality assured and pedagogically presentation of selected findings of major health relevance. In Norway, the first option is not permissible. Once a disclosure policy of returning individual results is introduced, the Norwegian Biotechnology Act will apply. Genetic testing is strictly regulated under this law with a demand for counselling and follow-up of positive predictive or pre-symptomatic test results. In addition the Health Research act demands that if individual information is to be disclosed, researchers are obliged to present it in a comprehensible way to the participant [12].

If the genetic test is of a predictive nature, a demand of pre-counselling is written into the law [13]. Legal professionals in The Norwegian Health Inspectorate have already considered whether whole genome sequencing should be classified as a predictive genetic test and their conclusion was positive. Genetic testing, they argued, should be judged on the possible consequences of the test and not on the intention of the test, and since predictive information is a possible consequence of whole genome sequencing, the test is a predictive test according to legal professionals in Norway [17]. Genetic counselling "before, during and after the test" is required for a predictive genetic test in Norway according to the law [13].

A disclosure policy in the HUNT biobank, then, will probably mean practical changes like this: New consents need to be obtained in order to inform participant about the use of whole genome sequencing of their samples as well as what the actual disclosure policy entails. When new consents are obtained the participants will need to be counselled about possible consequences of the whole genome information (or data generated by whole genome sequencing) and what kind of incidental findings that may arise. Then participants must take a decision regarding whether to participate or not, and whether they want to have incidental findings returned or not. In addition, the participant will be informed about their right to access their sequence on request.

Several practical and theoretical challenges arise: What does genetic counselling mean in a situation where there is no disease running in the family and nothing to counsel on? What kind of incidental findings, on a never ending list of genetic mutations, should be used to illustrate an incidental finding? What type of genetic information should be given as feedback and what should not be disclosed? Who is qualified to do counselling based on whole genome data? Are there enough professionals with the appropriate competence? What does genetic counselling and follow up of hundreds or thousands of participants in large scale biobanking cost? Who should pay for widespread counselling and re-testing and quality assurance of research data?

These questions are hard to answer, but an even harder question must be addressed: How does reconsent, genetic counselling and the weighing for or against return of results, affect the expectations, the concerns and the weal and woe of individual participants? The answer to those questions is that we don't know. But a disclosure-policy combined with reconsents, pre-counselling and in general a comprehensive focus on genetics, runs a risk of leading to harmful consequences. Presumably healthy persons might become preoccupied with genetics, with the "secrets" that they think are hidden in the sequence, with information about increased risk of future diseases, with individual prevention possibilities and with questions about reproductive risks. They will probably demand follow-up programs to handle their risks for future diseases, testing of family members, increased prenatal diagnostics, and so on.

The irony then is that the same researchers and research institution that wanted to combat the increasing medicalization of individuals in our society by their public health research, risk ending up with a more thorough medicalization of the local community than they ever had imagined. This might be the consequence, not because the researchers support such a development, but because "good ethics" demands it. A huge biobank and epidemiological study runs the risk of being transformed into a population based clinic for genetic counselling. In addition it might reduce the intrinsic value of research when individuals that solely would like to contribute to the "greater good" no longer have this opportunity.

In our view this is a failure of what could be labelled an "individualistic" research ethics, that has become, to use Dawson and Verweijs word, "too much inclined to assume the priority of individual autonomy, and too little interested in concepts such as 'public' and 'common', and what we share as fellow citizens or human beings" [18]. What's wrong with such an ethic is the supposition of an omnipresent antagonism between the interest of researchers and the interests of research participant. In that perspective, more rights for research participant, more choices, more access to data, more consent and more return of results will always be more of something which is good per se. It is a research ethic that fails to see the potential harmful public health consequences of its own practice. It fails to respect donor's intention of taking part in research and not clinical care. It reduces the potential research outcome by its enormous economical cost and thereby undermines the reason why individuals should take part in research. It is a research ethic that may lead to unethical consequences.

\section{THE RESCUE PRINCIPLE}

Having claimed that population based biobank research is not clinical care, and that believing so may lead to unethical consequences, still the question of disclosure of incidental findings won't go away. We still seem to struggle with some kind of moral intuition that we have a duty to save lives whenever we can. It doesn't matter 
whether we are just "pure" researchers or we are doctors in a doctor-patient relation. Indeed, no one has a moral duty to be a moral hero (for instance put his own life at risk in order to rescue a stranger), but if we can save lives by only making a "slight sacrifice" (for instance re-identify a sample and re-contact a participant) then we seem to have a moral duty to save lives.

This intuition is often referred to as the "rescue principle". Thomas Scanlon has formulated as follows: "If we can prevent something very bad from happening to someone by making a slight or even moderate sacrifice, it would be wrong not to do so" [19]. In other words: If I can easily save a baby that is about to drown, it's my duty to do so.

The rescue principle is a good reason for accepting that disclosure of incidental findings sometimes can be the only right thing to do. If a researcher "stumbles" over certain findings in his analysis, indicating that the research participant soon may die if he is not given medical treatment, this seems to represent an obligation to act on the information. The "sacrifice" that the researcher has to make in order to inform, must be regarded as "slight" and to avoid death must be said to be preventing something very bad from happening.

The question, however, in population based genetic research, is whether it is likely that such a situation will occur. As Forsberg et al claim, conditions leading to scenarios above do not characterize large-scale biobank research at all [10]. Genetic risk is seldom acute. The most common example of incidental findings in popular debates on genetics is the finding of a mutation in the BRCA1 or BRCA2 genes. In this case, we face genetic mutations with about $60-90 \%$ penetrance [21-23]. We don't know the time of onset of the potential disease and we don't know how a particular patient would respond to treatment. At the same time we do know that a positive test may cause painful decisions regarding removing or not removing healthy breasts in persons at risk. So even in this "paradigmatic" case for disclosure (according to the proponents of disclosure), we are very far from a situation similar to the situation of preventing a baby from drowning. By disclosing a mutation in BRCA1 or 2 we cannot be really sure whether we prevent something very bad from happening or whether we cause something bad to happen. By preventing a baby from drowning, we can be absolutely sure that we prevent something very bad from happening.

But even if we admit that the rescue principle, at least in principle, could be valid also for population based biobanking, there is no need to change the whole policy and regulation of this type of research to a system of disclosure, counselling and choice. If the rescue principle is valid, then it is valid in all types of research where humans are involved from social and behavioral science, via basic research to complex clinical trials. Research participants do not need to be counselled in advance in order to know whether they want to be saved or not according to the rescue principle. When we can avoid something very bad from happening, like rescue the baby that is about to drown, we don't ask the baby first whether it will be rescued or not.

\section{THE NORMATIVE STRUCTURE OF PROFESSIONAL RELATIONSHIP}

We have said that the rescue principle is valid in biobank research as in all other areas of life, but we doubt that it would be applicable in research utilizing whole genome sequencing. Firstly, genomic information that has significant "immediate lifesaving clinical utility" might not be found often. Secondly, because the most detrimental genetic changes probably will be the rare events that are unique for you or just a few of your family members and the deciphering of this information will not take place in a research setting. Thirdly, for more common serious genetic changes clinical genetic testing will be available and this will be the appropriate arena for testing and disclosing genetic information.

We have further claimed that population based biobank research is far from a clinical context and that the duties and rights from the clinical context are not valid for this research context.

A last objection to our non-disclosure policy must be launched. Even though researchers are not necessarily doctors and are not part of a doctor-patient relationship, the question remains whether there are relevant aspects of the researcher-participant relationship that still are unaddressed? Could there be anything in this relationship that implies that researchers owe more to participants than just trying to maximize the research output from the donation or rescue the life of a participant if he or she is in great danger?

Miller et al made an analogy that, in their opinion, "forces" the researcher to disclose incidental findings. Their analogy goes like this:

"A plumber asked to make a repair in a homeowner's basement might detect subtle but serious signs of termite infestation. This incidental finding involves access to private information - the plumber has no right to observe the condition of the homes where he works without the homeowners' consent. The plumber could take the stance that such incidental findings, being outside the scope of contracted work, are none of his business. However, in light of his professional relationship to the homeowner, his consensual access to private information and observations about the home, and his superior competence to recognize the termite problem, the plumber has thus an obligation to inform the homeowner of the problem." [20]

What Miller et al are saying is that the researcherparticipant relation is a professional relationship in the same way as a plumber-homeowner-relation. The superior competence of the plumber, or the researcher, in recognizing problems, means that a plumber or a researcher that just closes his eyes to incidental findings outside the "contracted work", is not acting professio- 
nally. This means that even though we don't have a doctor-patient relationship in population based biobank research, disclosure should be the rule, says Miller et al, because we have a professional relationship.

However, while Miller et al's analogy has a bearing in what we could term as "therapeutic research" it fails in many respects when applied to population based biobank research. First, the homeowner has invited the plumber in order to repair the pipes in his house. He can reasonably expect that he should be informed about a termite infestation, since a termite infestation will soon lead to a need for new repairs in the homeowner's house, and the whole point of having a plumber in his house is to repair the house. In population based biobank research it is the participants that are invited to take part in research, not the other way around. In addition they are not invited with the aim of having something repaired.

Second, a premise for talking about a professional relationship between a plumber and a homeowner seem to be that it actually involves a relationship. If the plumber is withholding information about a termite infestation he is withholding information for a John or a Laura. He is in their house, talking to them, or writing to them and finally he sends the invoice to them. All this time he relates to John or Laura and they relate to him, and this relationship is a central basis for certain expectations of openness, trust and disclosure. This is not very different from the expectations of openness, trust and disclosure that one may have in a doctorpatient relationship.

But such a relationship is present only in the recruitment phase of a biobank where samples and data are collected. In such a phase, feedback on blood pressure, cholesterol level, and other "immediate" data, might be given, precisely because it seem to be obvious as part of the face to face relation which exist at that time. An element of this professional relation is to inform the participants that from no on they will not relate any more. By coding the samples, promising anonymity and protecting against re-identification, the biobank has actually said that the professional relation between future researchers and the participants is a non-relation. The normative structure of this relation cannot then be an argument for disclosing incidental findings.

As we have seen, Miller et al's analogy fails to provide the ground for more comprehensive duties towards research participants in population based biobank research. That a plumber, due to his professional relationship, should inform a customer about a termite infestation, does not imply that a researcher in population based biobank research, due to his professional relationship, should tell a participant about incidental findings in general. Only when we change the context of research to a clinical one, where relationships have a certain meaning, and where participants are patients with a reasonable expectations to benefit from research, their analogy becomes relevant.

\section{CONCLUSION}

In this paper the question of disclosure of incidental findings in medical research has been discussed with regards to what researchers and research institutions owe research participants. We used two radically different context, patient near clinical research and population based biobank research, in order to demonstrate that what researchers owe research participants depends on the nature of the research. In therapeutic research which resembles a doctor-patient relation, the duty to inform, to disclose and to counsel is paramount. Such a duty however, is not transferable to large scale population based biobank research. What researchers and research institutions primarily owe research participants in this arena is to maximize the research output of their donation. A non-disclosure policy in large scale population based biobanks does not harm anyone, does not make anyone worse off, does not treat anyone like a mere mean, does not conflict with the normative structure of a professional relationship and is fully compatible with central virtues including respect, justice, reciprocity and beneficence in research.

Further, we posit that a disclosure policy in large scale biobank research may have harmful public health consequences in the sense that it could trigger and stimulate an excessive occupation with genetic risk and potential future disease in individuals that originally perceived themselves to be healthy. A disclosure policy runs the risk of contributing to a further medicalization of healthy individuals. In addition, it runs the risk of substantially reducing the public benefit of research because so many resources need to be transferred to individual counselling, information and follow up. In such a situation, where no moral principle or value demands disclosure and where possible consequences of disclosure could be really harmful, our conclusion is that a non-disclosure policy is the most ethical policy for large scale population based biobanking today.

Having said this, we need to add that a nondisclosure policy is not synonymous with a defence of secrecy, lack of information and non-transparency in research. Researchers have a duty to inform about their projects, about their hypothesis and about their results. The point is that such duties should be understood at a collective level - in line with the nature of public health research - and not as clinical obligations aimed at intervening in the life of individuals. We have also stressed that in population based biobank research, as in every other area of life, the rescue principle is valid. Based on this principle, a duty to disclose is always a theoretical possibility. We have, however, doubted that the rescue principle is of much relevance for the analyses being conducted in biobank research. Anyway, exceptional actions based on the rescue principle do not mean that population based biobanking should in general change their non-disclosure policy.

Between our two extreme points - large scale population based biobank research on the one hand and 
therapeutic research/clinical diagnostics on the other hand - there will be many different applications of whole genome sequencing in research. It's outside the scope of this paper to give recommendations for all imaginable projects on the axes between the two. However, by presenting these two extremes, we have presented a heuristic device for approaching the question of incidental findings and individual return of results that can be taken further by research ethical committees, internal ethical review boards and local wisdom. Our rule of thumb is simple: As research moves closer to the clinical setting, the correctness and relevance of a disclosure policy increases. Likewise, the further away research is from the clinical setting, the more increases the relevance and the correctness of a non-disclosure policy.

\section{REFERENCES}

1. Wolf SM, Lawrenz FP, Nelson CA, Kahn JP, Cho MK, Clayton EW, et al. Managing incidental findings in human subjects research: analysis and recommendations. J Law Med Ethics 2008; 36: 219-248.

2. Jonas H. Technik, Medizin und Ethik. Zur Praxis des Prinzips Verantwortung. Suhrkamp, 1987.

3. Ravitsky V, Wilfond BS. Disclosing individual genetic results to research participants. Am J Bioeth 2006; 6: 8-17.

4. Meltzer LA. Undesirable implications of disclosing individual genetic results to research participants. Am $J$ Bioeth 006; 6 (6): 28.

5. Parker LS. Letting the gene out of the bottle: a comment on returning individual research results to participants. Am J Bioeth 2006; 6 (6): 22.

6. Parker LS. The future of incidental findings: should they be viewed as benefits? J Law Med Ethics 2008; 36: 341-351.

7. Cho MK. Understanding incidental findings in the context of genetics and genomics. J Law Med Ethics 2008; 36: $280-285$.

8. Wade $\mathrm{CH}$, Kalfoglou AL. When do genetic researchers have a duty to recontact study participants? Am $J$ Bioeth 2006; 6 (6): 26.

9. Ossorio PN. Letting the gene out of the bottle: a comment on returning individual research results to participants. Am J Bioeth 2006; 6 (6): 24.

10. Forsberg JS, Hansson MG, Eriksson S. Changing perspectives in biobank research: from individual rights to concerns about public health regarding the return of results. Eur J Hum Genet 2009; 17: 1544-1549.

11. WMA Declaration of Helsinki - Ethical Principles for Medical Research Involving Human Subjects. 2008, http://www.wma.net/en/30publications/10policies/b3/.

12. ACT 2008-06-20 no. 44: Act on medical and health research (the Health Research Act).

13. Act of 5 December 2003 No. 100 relating to the application of biotechnology in human medicine, etc.

14. The Norwegian Biotechnology Advisory Board. Nordmenns arvestoff fellesskapsressurs og personlig informasjon. Letter to the Ministry of Health and Care Services, 20.12.2010.

15. Carlsen T. HUNT - 25 år for folkehelsen. Universitetsavisa, 03.08.2009.

16. Getz L, Kirkengen AL, Hetlevik I, Romundstad S, Sigurdsson JA. Ethical dilemmas arising from implementation of the European guidelines on cardiovascular disease prevention in clinical practice. A descriptive epidemiological study. Scand J Prim Health Care 2004; 22 (4): 202-8.

17. Genomanalyse i forbindelse med klinikknære prosjekter. Letter from the Norwegian Directorate of Health, 22.12.2010.

18. Dawson A, Verweij M. Public health ethics: A manifesto. Public Health Ethics 2008; 1 (1): 1-2.

19. Scanlon T. What We Owe to Each Other. Cambridge, MA: Harvard University Press, 1998.

20. Miller FG, Mello MM, Joffe S. Incidental findings in human subjects research: what do investigators owe research participants? J Law Med Ethics 2008; 36: 271-279.

21. Warner E, Foulkes W, Goodwin P, Meschino W, Blondal J, Paterson C, et al. Prevalence and penetrance of BRCA1 and BRCA2 gene mutations in unselected Ashkenazi Jewish women with breast cancer. $J$ Natl Cancer Inst 1999; 91 (14): 1241-1247.

22. Evans DG, Shenton A, Woodward E, Lalloo F, Howell A, Maher E. Penetrance estimates for BRCA1 and BRCA2 based on genetic testing in a Clinical Cancer Genetics service setting: Risks of breast/ovarian cancer quoted should reflect the cancer burden in the family. BMC Cancer 2008; 8 (1): 155.

23. Heimdal K, Maehle L, Apold J, Pedersen JC, Møller P. The Norwegian founder mutations in BRCA1: high penetrance confirmed in an incident cancer series and differences observed in the risk of ovarian cancer. Eur $J$ Cancer 2003; 39 (15): 2205-2213. 